\title{
Evaluation of atlas-based segmentation of hippocampi in healthy humans
}

Authors:

Roman Rodionov $^{1,2}$, Marie Chupin ${ }^{3 *}$, Elaine Williams ${ }^{2}$, Alexander Hammers ${ }^{1,4}$, Chandrasekharan Kesavadas ${ }^{5}$, Louis Lemieux ${ }^{1,2}$

Affiliations:
${ }^{1}$ Department of Clinical and Experimental Epilepsy, UCL Institute of Neurology, Queen Square, London WC1N 3BG, United Kingdom

${ }^{2}$ MRI Unit, National Society for Epilepsy, Chalfont St Peter, Buckinghamshire SL9 0RJ, United Kingdom

${ }^{3}$ Cognitive Neuroscience and Brain Imaging, CNRS UPR640, UPMC Paris6, Paris, France

${ }^{4}$ Department of Clinical Neuroscience, Division of Neuroscience and Mental Health, and MRC Clinical Sciences Centre, Imperial College London, London W12 0NN, United Kingdom

${ }^{5}$ Sree Chitra Tirunal Institute for Medical Sciences and Technology, Trivandrum, India

* These two authors contributed equally

Corresponding author:

Roman Rodionov: r.rodionov@ion.ucl.ac.uk 


\begin{abstract}
Introduction \& Aim: Region of interest (ROI) based fMRI data analysis relies on extracting signals from a specific area which is presumed to be involved in the brain activity being studied. The hippocampus is of interest in many functional connectivity studies $[1,2]$ for example in epilepsy as it plays an important role in epileptogenesis. In this context, ROI may be defined using different techniques. Our study aims at evaluating the spatial correspondence of hippocampal ROIs obtained using three brain atlases with hippocampal ROI obtained using an automatic segmentation algorithm dedicated to the hippocampus.
\end{abstract}

Material \& Methods: High-resolution volumetric T1-weighted MR images of eighteen healthy volunteers (five females) were acquired on a 3T scanner. Individual ROIs for both hippocampi of each subject were segmented from the MR images using an automatic hippocampus and amygdala segmentation software called SACHA [3] providing the gold standard ROI for comparison with the atlas-derived results. For each subject, hippocampal ROIs were also obtained using three brain atlases: PickAtlas available as a commonly used software toolbox [4,5]; AAL (Automated Anatomical Labeling) atlas [6] included as a subset of ROI into PickAtlas toolbox; a frequency based brain atlas by Hammers et al [7]. The levels of agreement between the SACHA results and those obtained using the atlases were assessed based on quantitative indices measuring volume differences and spatial overlap. The comparison was performed in standard MNI space, the registration being obtained with SPM5 (http://www.fil.ion.ucl.ac.uk/spm/).

Results: The mean volumetric error across all subjects was $73 \%$ for hippocampal ROIs derived from AAL atlas; $20 \%$ in case of ROIs derived from the Hammers atlas; $107 \%$ for ROIs derived from Pickatlas. The mean false positive and false negative classification rates were $60 \%$ and $10 \%$ respectively for the AAL atlas; $16 \%$ and $32 \%$ for the Hammers atlas; $6 \%$ and $72 \%$ for the PickAtlas. Conclusion: Though atlas-based ROI definition may be convenient, the resulting ROIs may be poor representations of the hippocampus in some studies critical to under- or over-sampling. Performance of the AAL atlas was inferior to that of the Hammers atlas. Hippocampal ROIs derived from PickAtlas are highly significantly smaller, and this results in the worst performance out of three atlases. It is advisable 
that the defined ROIs should be verified with knowledge of neuroanatomy before before using it for further data analysis.

Key words: region of interest, hippocampus, segmentation, brain atlas 


\section{Introduction}

Functional connectivity as one of the methods of ROI-based analysis of fMRI data includes step of extracting BOLD signal from a specified ROI $[8,9,10,11,12,13]$. Given the functional and anatomical parcelation of the brain, the shape of the ROI is very important for ensuring that the area of interest is fully covered, and that voxels belonging to neighbouring areas are excluded as they might be either from a different functional brain areas or from an area which can not produce BOLD signal (e.g. white matter or CSF). Two most widely used ways of specifying ROI are: (1) Individually segmented ROI using manual segmentation performed by an expert-neuroanatomist or by an automatic algorithm performed by a specific software, for example [3]; (2) Atlas-based ROI. The brain atlases employed in fMRI studies are created in a standard space (Talairach or MNI). The description of possible methodologies applied to build brain atlases can be found in [14]. Since there is a substantial variability in the macroscopic anatomy between individuals, the best practice is to define ROI for each subject based on their own anatomy. However, most studies use atlas derived ROI and hence it becomes necessary to evaluate these ROI derived from atlases compared with those derived from individual anatomy.

To the best of our knowledge there has been no study investigating sensitivity of the results of functional connectivity studies with respect to the shape and volume of the ROI used to sample brain areas of interest. Here we investigate one aspect of this issue: the variance of the shape and volume of the hippocampal ROI derived from three brain atlases: a frequency based brain atlas by Hammers et al [7] and two more widely used, single-subject atlases: AAL [6] and Brodmann areas defined in the PickAtlas toolbox $[4,5]$. In this study we used the extended version of the frequency atlas [7] based on manual delineations of 30 brains. The maximum probability map was obtained after co-registering all individual atlases into MNI space using the "Segment" module in SPM5 (http://www.fil.ion.ucl.ac.uk/spm/). We compare the atlas-derived ROIs with the results of the segmentation using an automatic algorithm, SACHA, implemented as part of the Brainvisa environment (http://brainvisa.info) [15]. 
Our interest to evaluate hippocampal ROI is explained by the importance of this structure in studies (especially ROI-based functional connectivity analisys of fMRI data) in patients with epilepsy and Alzheimer's disease $[1,2,16,17,18]$. 


\section{Materials \& Methods}

\section{Data}

Eighteen healthy subjects (five females; mean age 34.7 years and range: 25 - 56 years) were included. The criterion for inclusion into the study was absence of neurological pathology. All subjects gave written informed consent (Joint Ethics Committee of the National Hospital for Neurology and Neurosurgery and UCL Institute of Neurology).

High-resolution 3D T1-weighted MR images were acquired (Fast Spoiled Gradient Recalled [FSPGR]) on a 3T General Electric Excite HD scanner using a standard head coil: TR/TE/TI 8/3.1/450ms, flip angle $20^{\circ} ; 1561.1 \mathrm{~mm}$-thick coronal slices; matrix $256 \times 256 ; 24 \times 18 \mathrm{~cm}$ field of view; scan time 7 min.

\section{Data processing and analysis}

Individual ROIs for both hippocampi of each subject were segmented from the MR images using SACHA. The comparison of the atlas-based ROIs and individual SACHA-derived ROIs (further called as "individual ROIs") was performed in MNI space for two reasons: the fMRI analyses were to be performed in MNI space; all three atlases are available in MNI space. SACHA ROIs were evaluated by a trained observer (EW) for ensuring their consistency as a gold standard. T1-weighted images were transformed to MNI space using nonlinear warping as implemented in SPM5 [19]. The resulting transformation parameters were applied to the images of individual ROIs in order to register them to MNI space (voxel size in the template image is $2 \times 2 \times 2 \mathrm{~mm}$ ). The result of the registration was checked in the area of both hippocampi by visual evaluation by an expert-neuroanatomist (CK).

Using individual ROIs as the gold standard, the following volumetric and spatial correspondence measures were calculated as described in $[20,21]: \mathrm{RV}=$ the relative error on volume (the optimal value is $0 \%$ ); $\mathrm{K}=$ Dice overlap index, quantifying the proportion of properly classified voxels (the optimal value is $100 \%$ ); FP and $\mathrm{FN}=$ the proportions (in $\%$ of total $\mathrm{ROI}$ volumes) of false positive and false negative voxels according to SACHA-based ROIs, respectively. In addition, the distance between the centre of the surface voxels of two ROIs is considered in three ways (indices measured in millimeters): the average symmetric distance on the whole boundary, Dm; the maximum of 
the symmetric distance (Hausdorff distance), DM; 95 percentile of DM, D95. The formulas for the indices can be found in the Appendix.

Two tailed t-test has been performed to test difference between mean values of the calculated indices comparing performance of (1) different atlases and (2) performance of right and left ROI within each atlas. 


\section{Results}

The results in the form of summary statistics of the calculated indices (mean, standard deviation, minimum and maximum values) are summarised in the table 1. Individual ROIs obtained for right hippocampus of subject \#8 overlaid over atlas-based ROIs are shown separately for each atlas on the same slices (Figure 1).

The values of the index describing the averaged similarity of the shape of the individual and atlas-derived ROI (Dm, in mm) show better performance of the Hammers atlas: for the right ROIs, AAL atlas $3.5(\mathrm{SD}=0.3)$, Hammers atlas $2.6(\mathrm{SD}=0.7)$, PickAtlas $3.5(\mathrm{SD}=0.4)$; for the left ROIs, AAL atlas $3.7(\mathrm{SD}=0.5)$, Hammers atlas $2.8(\mathrm{SD}=0.6)$, PickAtlas $3.6(\mathrm{SD}=0.9)$. However the values of the index describing the extreme deviation of the shape of the individual and atlas-derived ROI (DM, in $\mathrm{mm}$ ) show that the largest local error is to be found for Hammers atlas: for the right ROIs, AAL atlas 12 $(\mathrm{SD}=2)$, Hammers atlas 15.3 ( $\mathrm{SD}=2)$, PickAtlas $11(\mathrm{SD}=1.7)$; for the left ROIs, AAL atlas $12(\mathrm{SD}=1)$, Hammers atlas $16(\mathrm{SD}=2)$, PickAtlas $11.3(\mathrm{SD}=2.2)$.

Both two tailed t-test and non-parametric Wilcoxon rank test performed to reveal difference between mean values of the indices calculated for ROI from the three atlases showed highly significant $(p<0.001)$ difference between mean values of the indices RV, K, FP, FN both for right and left ROI. There is no significant $(\mathrm{p}<0.001)$ difference between mean values of the indices Dm, DM, D95 for AAL atlas and PickAtlas (both for right and left ROI), whereas significant $(\mathrm{p}<0.001)$ difference is revealed for Hammers atlas compared with both other atlases.

A significant difference $(\mathrm{p}<0.01)$ between mean values for right and left ROI has been observed in the following indices: FN index for AAL atlas; RV, FP, FN indices for PickAtlas. No such difference was observed for any index for the Hammers atlas. 


\section{Discussion}

Using T1-weighted brain images from eighteen healthy volunteers, we performed spatial comparison between individually segmented hippocampal ROIs (done using software SACHA and checked by an expert as suitable to be considered as gold standard) and ROI derived from three brain atlases: Hammers et al's frequency based atlas, AAL atlas and the Brodmann areas available in the PickAtlas toolbox.

The AAL atlas contains ROIs defined manually on the high resolution MNI single-subject MRI brain template [6]. The PickAtlas [5] uses the MNI template for normalisation and probes the Talairach Daemon [4] across the entire Talairach space (created from a single hemisphere of a single subject) to generate tables based on coordinate position. In contrast, the frequency based atlas used in this work [7] was developed using multiple subjects in stereotaxic space. After manual segmentation in the individual space, the MRI volumes of the subjects were spatially normalised to T1-weighted MRI template in MNI/ICBM 152 space, as contained in the SPM5 package. This significant difference in the approaches applied to develop the three atlases chosen for this study along with differences in manual segmentation protocols used to define ROIs explain better volumetric correspondence of the individual ROIs and ROIs derived from the frequency based atlas (indices RV, K, FP), although with a greater false negative rate.

The three atlases chosen for our study rely on normalization of the individual brain to a stereotaxic space, a process that could contribute to the degradation of the segmentation results. Therefore, the high degree of spatial correspondence of the normalised individual T1-weighted images and MNI template in the area of both hippocampi was confirmed by an expert-neuronatomist after visual evaluation, which should always form part of ROI-based fMRI data analyses.

When taking into account the results of the comparison of ROIs performed in this study in the context of an ROI-based analysis of fMRI data, it is necessary to note that the comparison was performed in the space of the T1-weighted images warped to the MNI space. The fMRI data has to be warped to the same space in order to use both individual and atlas-based ROIs. The uncertainty of warping fMRI data has to be considered when specifying the required precision for the definition of ROIs. For example, in the case of hippocampal ROIs the fMRI data is prone to severe distortions in the 
area of interest causing increased uncertainty in the result of warping which may have a greater influence on the quality of the results than ROI definition. In this context it may appear that the frequency based atlas used in our study provides hippocampal ROIs with accuracy which is satisfactory for most studies as the observed difference in comparison with individual ROI (indices RV, K, FP, FN, $\mathrm{Dm})$ is of the same level of magnitude as the effect of uncertainties introduced while warping the fMRI data from individual to MNI space using up to date methods [22]. It is most likely that special investigation is necessary to conclude the same about hippocampal ROIs derived from PickAtlas and AAL atlas, as the accuracy of segmentation is very low (Table 1).

The low performance of Hammers atlas compare to other two atlases in DM and D95 indices is due to significant underestimation of the hippocampal tail which is detected by visual comparison of individual ROIs and ROIs derived from atlases.

The absence of the difference between mean values of the indices for right and left ROI observed in Hammers atlas as oppose to two other atlases (see Results) is indicative of better performance for the Hammers atlas and may reflect the fact that AAL atlas and PickAtlas are single-brain atlases and therefore are more subject to this type of bias.

In spite of the extreme underestimation, the ROI from PickAtlas might be satisfactory for the studies in which overestimation has to be avoided; in fact, false positive ration is lower for PickAtlas than for the other two atlases.

PickAtlas uses nonlinear transformation [23] to convert coordinates between MNI and Talairach spaces [5]. More precise transformation was suggested recently [24], which suggests that the accuracy of ROIs generated by PickAtlas toolbox may be increased.

This study was performed using data obtained from healthy volunteers and the results can not be extrapolated to cases with pathology or abnormal brains. However, one should generally assume that, in patients with hippocampal abnormalities (e.g. hippocampus sclerosis), the performance of any atlas will be much worse than in our study and that the levels of performance obtained here represent upper bounds. Therefore using individual segmentation (either automated or manual) is advisable in pathological cases. 


\section{Future work}

The sensitivity of functional connectivity estimates to ROI definition methods remains to be investigated. Our results suggest that ROI definition methodology can have a drastic influence on fMRI studies of hippocampal activity, with even greater impact in pathological cases. This highlights the direction for further work. 


\section{Conclusions}

The frequency based atlas [7] demonstrates higher accuracy for hippocampal segmentation than AAL atlas and PickAtlas in healthy volunteers. We recommend that the inclusion of erroneously classified voxels and exclusion of erroneously unclassified voxels must be carefully evaluated and measures taken

to minimise their impact on the sensitivity and specificity of the correlation studies (specifically ROIbased functional connectivity studies using fMRI data). 


\section{Acknowledgements}

This work was undertaken at UCLH/UCL who received a proportion of funding from the UK

Department of Health's NIHR Biomedical Research Centres funding scheme. Work funded through a grant from the Medical Research Council (MRC grant number G0301067). We are grateful to the Big Lottery Fund, Wolfson Trust and National Society for Epilepsy for supporting the NSE MRI scanner.

CK was funded through the Biotechnology associateship programme of the Department of Biotechnology, Government of India. 


\section{References}

1. Wagner K, Frings L, Halsband U, Everts R, Buller A, Spreer J, Zentner J, SchulzeBonhage A. Hippocampal functional connectivity reflects verbal episodic memory network integrity. Neuroreport. 2007;18(16):1719-23.

2. Bettus G, Guedj E, Joyeux F, Confort-Gouny S, Soulier E, Laguitton V, Cozzone PJ, Chauvel P, Ranjeva JP, Bartolomei F, Guye M. Decreased basal fMRI functional connectivity in epileptogenic networks and contralateral compensatory mechanisms. Hum Brain Mapp. 2008; [in press]

3. Chupin M, Hammers A, Bardinet E, Colliot O, Liu RS, Duncan JS, Garnero L, Lemieux L. Fully automatic segmentation of the hippocampus and the amygdala from MRI using hybrid prior knowledge. Med Image Comput Comput Assist Interv Int Conf Med Image Comput Comput Assist Interv. 2007;10(Pt 1):875-82

4. Lancaster JL, Woldorff MG, Parsons LM, Liotti M, Freitas CS, Rainey L, Kochunov PV, Nickerson D, Mikiten SA, Fox PT. Automated Talairach atlas labels for functional brain mapping. Hum. Brain Mapp. 2000; 10 (3): 120-131.

5. Maldjian JA, Laurienti PJ, Kraft RA, Burdette JH. An automated method for neuroanatomic and cytoarchitectonic atlas-based interrogation of fMRI data sets NeuroImage 2003; 19: 1233-1239.

6. Tzourio-Mazoyer N., Landeau, B., Papathanassiou, D., Crivello, F., Etard, O., Delcroix, $\mathrm{N}$. Automated anatomical labeling of activations in SPM using a macroscopic anatomical parcellation of the MNI MRI single subject brain. Neuroimage 2002; 15 : 273-289

7. Hammers A, Allom R, Koepp MJ, Free SL, Myers R, Lemieux L, Mitchell TN, Brooks DJ, Duncan JS. Three-dimensional maximum probability atlas of the human brain, with particular reference to the temporal lobe. Hum Brain Mapp. 2003 ;19(4):224-47.

8. Bohm, C., Greitz, T., Seitz, R., Eriksson, L. Specification and selection of regions of interest (ROIs) in a computerized brain atlas. J. Cereb. Blood Flow Metab. 1991; 11: A64-A68.

9. Collins, D.L., Holmes, C.J., Peters, T.M., Evans, A.C.. Automatic 3-D model-based neuroanatomical segmentation. Hum. Brain Mapp. 1995; 3:190-208.

10. Yasuno, F., Hasnine, A.H., Suhara, T., Ichimiya, T., Sudo, Y., Inoue, M., Takano, A., Ou, T., Ando, T., Toyama, H. Template-basedmethod for multiple volumes of interest of human brain PET images. NeuroImage 2002; 16: 577-586.

11. Hammers A, Koepp MJ, Free SL, Brett M, Richardson MP, Labbé C, Cunningham VJ, Brooks DJ, Duncan J. Implementation and application of a brain template for multiple volumes of interest. Hum Brain Mapp. 2002 ;15:165-74.

12. Poldrack R A Region of interest analysis for fMRI. Soc Cogn Affect Neurosci. 2007; 2: 67-70 doi:10.1093/scan/nsm006

13. Buck R, Singhal H, Arora J, Schlitt H, Constable R.T Detecting change in BOLD signal between sessions for atlas-based anatomical ROIs NeuroImage 2008; 40: 11571165

14. Mazziotta JC. Brain mapping: its use in patients with neurological disorders. Rev Neurol. 2001; 157(8-9 Pt 1):863-71

15. Cointepas, Y., Mangin, J.-F., Garnero, L., Poline, J.-B., and, H. BrainVISA: software platform for visualization and analysis of multimodality brain data. HBM-2001, p. S98.

16. Zhang HY, Wang SJ, Xing J, Liu B, Ma ZL, Yang M, Zhang ZJ, Teng GJ. Detection of PCC functional connectivity characteristics in resting-state fMRI in mild Alzheimer's disease. Behav Brain Res 2008; [in press] 
17. Zhou Y, Dougherty JH Jr, Hubner KF, Bai B, Cannon RL, Hutson RK. Abnormal connectivity in the posterior cingulate and hippocampus in early Alzheimer's disease and mild cognitive impairment. Alzheimers Dement. 2008; 4(4):265-70.

18. Carmichael OT, Aizenstein HA, Davis SW, Becker JT, Thompson PM, Meltzer CC, Liu Y. Atlas-based hippocampus segmentation in Alzheimer's disease and mild cognitive impairment. Neuroimage. 2005 Oct 1;27(4):979-90

19. Ashburner J, Friston KJ. Unified segmentation. Neuroimage. 2005 Jul 1;26(3):839-51

20. Chupin M, Mukuna-Bantumbakulu A R, Hasboun D, Bardinet E, Baillet S, Kinkingnéhun S, Lemieux L, Dubois B, Garnerob L Anatomically constrained region deformation for the automated segmentation of the hippocampus and the amygdala: Method and validation on controls and patients with Alzheimer's disease Neurolmage 2007; 34 : 996-1019

21. Gerig, G., Jomier, M., Chakos, M., 2001. Valmet: a new validation tool for assessing and improving 3D image segmentation. MICCAI 2001, vol. 2208, pp. 516-523

22. Frackowiak, R.S.J., Friston, K.J., Frith, C., Dolan, R., Price, C.J., Zeki, S., Ashburner, J., Penny, W.D. Human Brain Function, 2nd edition. 2003 Academic Press

23. Brett M, Christoff K, Cusack R, Lancaster J. Using the Talairach atlas with the MNI template. NeuroImage 2001, 13:S85.

24. Lancaster JL, Tordesillas-Gutiérrez D, Martinez M, Salinas F, Evans A, Zilles K, Mazziotta JC, Fox PT. Bias between MNI and Talairach coordinates analyzed using the ICBM-152 brain template. Hum Brain Mapp. 2007 Nov;28(11):1194-205. 


\section{Appendix}

The segmentation quality indices compare the ROI from each atlas, Seg, with the standard ROI obtained by SACHA, Ref. Seven indices were used to quantify the accuracy of the method and facilitate comparison with published values [20,21].

$\mathrm{RV}$ is the relative error on volume; it expresses the difference in volume of segmented object

$\mathrm{O}_{\text {Seg }}$ and reference object $\mathrm{O}_{\text {Ref }}$, relatively to their average:

$$
R V\left(O_{\text {Seg }}, O_{\mathrm{Re} f}\right)=2 \frac{\left|V_{O_{\text {Seg }}}-V_{O_{\mathrm{Re} f}}\right|}{V_{O_{\text {Seg }}}+V_{O_{\mathrm{Re} f}}}
$$

The optimal value for this index, consistent with perfect agreement, is $0 \%$.

Index $\mathrm{K}$ characterises overlap between $\mathrm{O}_{\mathrm{Seg}}$ and $\mathrm{O}_{\mathrm{Ref}}$, describing the number of properly classified voxels, without taking into account the number of ill-classified voxels:

$$
K\left(O_{\text {Seg }}, O_{\operatorname{Re} f}\right)=2 \frac{V_{O_{S e g} \cap O_{\operatorname{Re} f}}}{V_{O_{S e g}}+V_{O_{\operatorname{Re} f}}}
$$

The optimal value is $100 \%$.

The numbers of false positives, FP, and false negatives, FN, are computed here relatively to the number of voxels labelled as $\mathrm{O}_{\text {Seg }}$ or $\mathrm{O}_{\text {Ref: }}$ :

$$
\begin{aligned}
& F P\left(O_{S e g}, O_{\operatorname{Re} f}\right)=\frac{V_{O_{S e g}}-V_{O_{S e g} \cup O_{\operatorname{Re} f}}}{V_{O_{\text {Seg }} \cap O_{\operatorname{Re} f}}} \\
& F N\left(O_{S e g}, O_{\operatorname{Re} f}\right)=\frac{V_{O_{\operatorname{Re} f}}-V_{O_{S e g} \cap O_{\operatorname{Re} f}}}{V_{O_{S e g} \cup O_{\operatorname{Re} f}}}
\end{aligned}
$$

The local behaviour on the boundary can be characterised by its surface voxels (defined as the voxels of $\mathrm{O}$ with at least one 26-neighbour outside of $\mathrm{O}$ ). The distance between the centre of the surface voxels of $\mathrm{O}_{\mathrm{Seg}}$ and those of $\mathrm{O}_{\mathrm{Ref}}$ is considered in three ways. First, the average symmetric distance on the whole boundary, Dm, is computed:

$$
\operatorname{Dm}\left(O_{\operatorname{Seg}}, O_{\operatorname{Re} f}\right)=\max \left[h\left(O_{\operatorname{Seg}}, O_{\operatorname{Re} f}\right), h\left(O_{\operatorname{Re} f}, O_{\operatorname{Seg}}\right)\right\rfloor
$$

where 
$h(A, B)=\frac{1}{N_{A}} \sum_{a \in A} d(a, B)$

Second, the maximum of the symmetric distance (Hausdorff distance), DM, is considered:

$D M\left(O_{\text {Seg }}, O_{\operatorname{Re} f}\right)=\max \left[H\left(O_{S e g}, O_{\operatorname{Re} f}\right), H\left(O_{\operatorname{Re} f}, O_{\operatorname{Seg}}\right)\right]$

where

$H(A, B)=\max _{a \in A}[d(a, B)]$

with d the Euclidian distance.

The last index is used to discard sporadic errors, by considering the distance which explains the 95 percentile of DM, and it was called D95. All distances are expressed in millimetres. 
Figure 1. Fragments of sagittal, coronal and axial projections of T1 weighted image showing individual ROI (subject 8) and ROI derived from (a) frequency based atlas, (b) AAL atlas, (c) PickAtlas. The same slices are shown in all three cases. The red colour in encodes voxels covered only by individual ROI, green - voxels covered only ROI from one of the atlases, yellow - voxels in the area of overlap between the individual ROI and ROI from an atlas. 
(a)
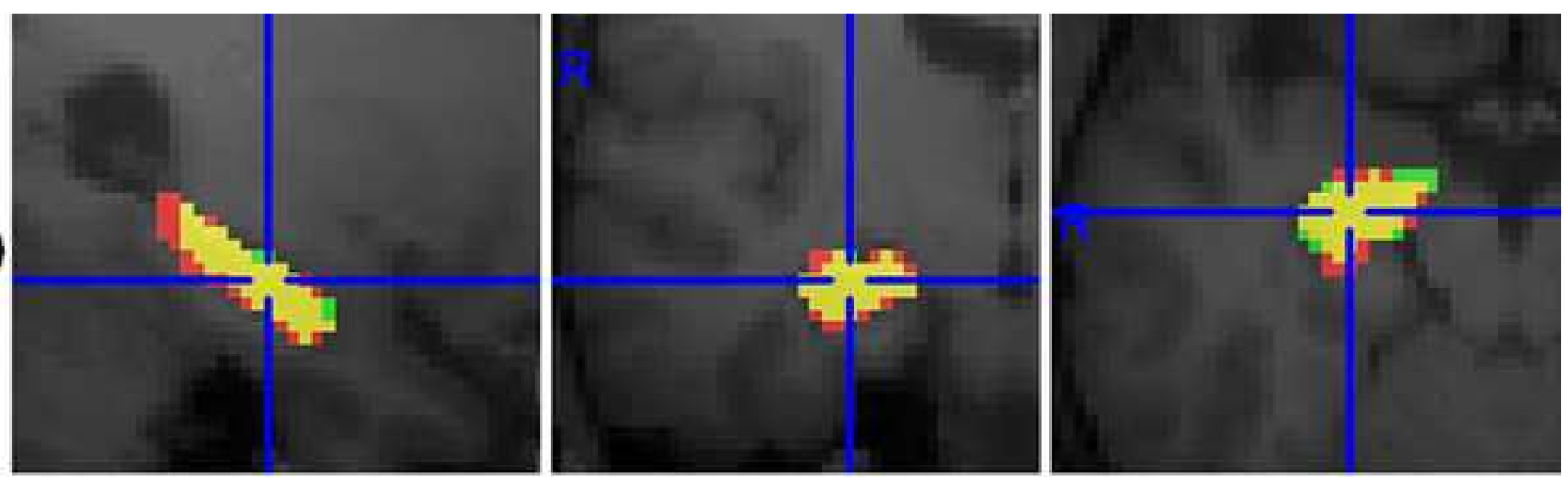

(b)
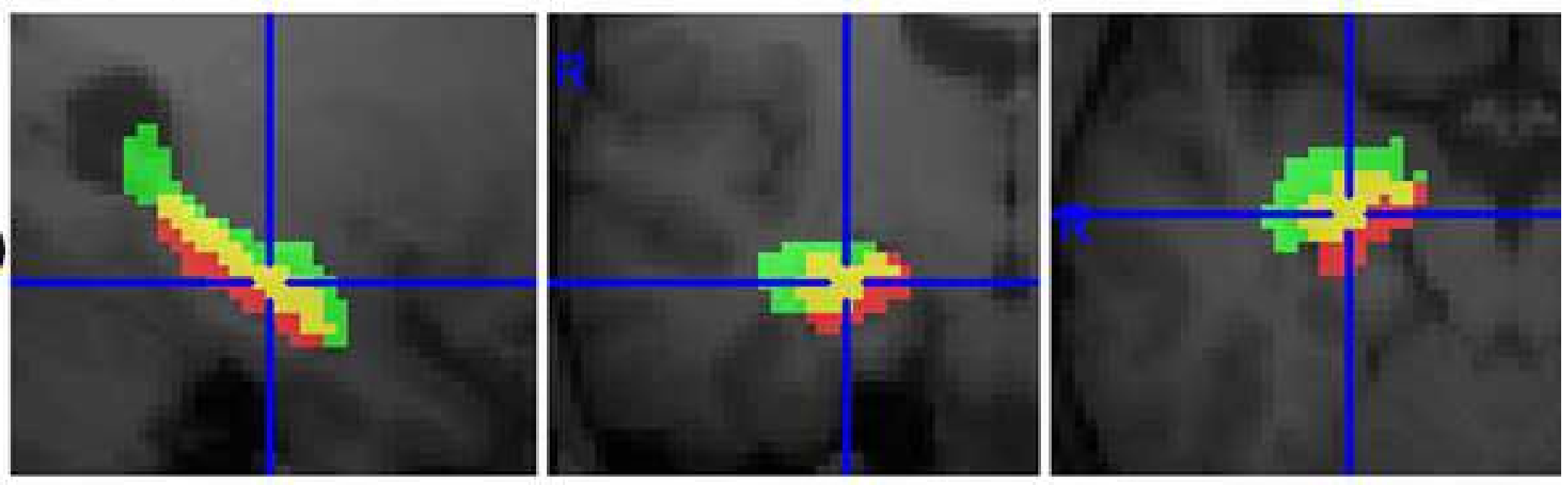

(c)
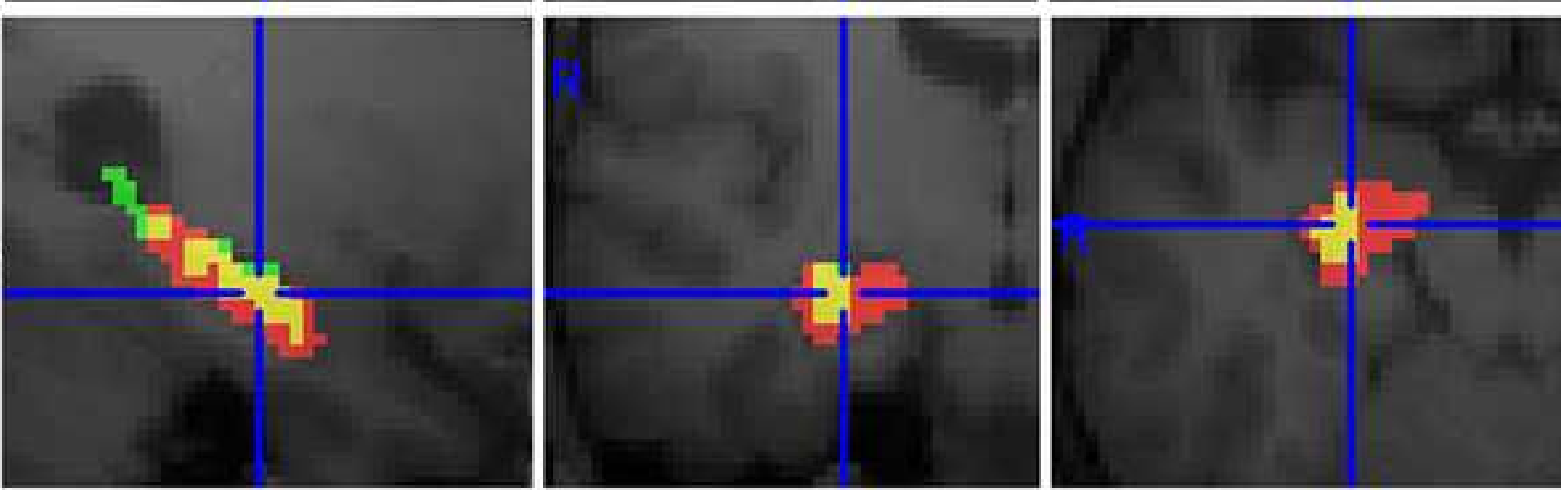


\section{Evaluation of atlas-based segmentation of hippocampi in healthy humans}

Authors:

Roman Rodionov $^{1,2}$, , Marie Chupin ${ }^{3 *}$, Elaine Williams ${ }^{2}$, Alexander Hammers ${ }^{1,4}$, Chandrasekharan Kesavadas ${ }^{5}$, Louis Lemieux ${ }^{1,2}$

\section{Affiliations:}

${ }^{1}$ Department of Clinical and Experimental Epilepsy, UCL Institute of Neurology, Queen Square, London WC1N 3BG, United Kingdom

${ }^{2}$ MRI Unit, National Society for Epilepsy, Chalfont St Peter, Buckinghamshire SL9 0RJ, United Kingdom

${ }^{3}$ Cognitive Neuroscience and Brain Imaging, CNRS UPR640, UPMC Paris6, Paris, France

${ }^{4}$ Department of Clinical Neuroscience, Division of Neuroscience and Mental Health, and MRC Clinical Sciences Centre, Imperial College London, London W12 0NN, United Kingdom

${ }^{5}$ Sree Chitra Tirunal Institute for Medical Sciences and Technology, Trivandrum, India

* These two authors contributed equally

Corresponding author:

Roman Rodionov: r.rodionov@ion.ucl.ac.uk 


\begin{abstract}
Introduction \& Aim: Region of interest (ROI) based fMRI data analysis relies on extracting signals from a specific area which is presumed to be involved in the brain activity being studied. The hippocampus is of interest in many functional connectivity studies $[1,2]$ for example in epilepsy as it plays an important role in epileptogenesis. In this context, ROI may be defined using different techniques. Our study aims at evaluating the spatial correspondence of hippocampal ROIs obtained using three brain atlases with hippocampal ROI obtained using an automatic segmentation algorithm dedicated to the hippocampus.
\end{abstract}

Material \& Methods: High-resolution volumetric T1-weighted MR images of eighteen healthy volunteers (five females) were acquired on a 3T scanner. Individual ROIs for both hippocampi of each subject were segmented from the MR images using an automatic hippocampus and amygdala segmentation software called SACHA [3] providing the gold standard ROI for comparison with the atlas-derived results. For each subject, hippocampal ROIs were also obtained using three brain atlases: PickAtlas available as a commonly used software toolbox [4,5]; AAL (Automated Anatomical Labeling) atlas [6] included as a subset of ROI into PickAtlas toolbox; a frequency based brain atlas by Hammers et al [7]. The levels of agreement between the SACHA results and those obtained using the atlases were assessed based on quantitative indices measuring volume differences and spatial overlap. The comparison was performed in standard MNI space, the registration being obtained with SPM5 (http://www.fil.ion.ucl.ac.uk/spm/).

Results: The mean volumetric error across all subjects was $73 \%$ for hippocampal ROIs derived from AAL atlas; $20 \%$ in case of ROIs derived from the Hammers atlas; $107 \%$ for ROIs derived from Pickatlas. The mean false positive and false negative classification rates were $60 \%$ and $10 \%$ respectively for the AAL atlas; $16 \%$ and $32 \%$ for the Hammers atlas; $6 \%$ and $72 \%$ for the PickAtlas. Conclusion: Though atlas-based ROI definition may be convenient, the resulting ROIs may be poor representations of the hippocampus in some studies critical to under- or over-sampling. Performance of the AAL atlas was inferior to that of the Hammers atlas. Hippocampal ROIs derived from PickAtlas are highly significantly smaller, and this results in the worst performance out of three atlases. It is advisable 
that the defined ROIs should be verified with knowledge of neuroanatomy before before using it for further data analysis.

Key words: region of interest, hippocampus, segmentation, brain atlas 


\section{Introduction}

Functional connectivity as one of the methods of ROI-based analysis of fMRI data includes step of extracting BOLD signal from a specified ROI $[8,9,10,11,12,13]$. Given the functional and anatomical parcelation of the brain, the shape of the ROI is very important for ensuring that the area of interest is fully covered, and that voxels belonging to neighbouring areas are excluded as they might be either from a different functional brain areas or from an area which can not produce BOLD signal (e.g. white matter or CSF). Two most widely used ways of specifying ROI are: (1) Individually segmented ROI using manual segmentation performed by an expert-neuroanatomist or by an automatic algorithm performed by a specific software, for example [3]; (2) Atlas-based ROI. The brain atlases employed in fMRI studies are created in a standard space (Talairach or MNI). The description of possible methodologies applied to build brain atlases can be found in [14]. Since there is a substantial variability in the macroscopic anatomy between individuals, the best practice is to define ROI for each subject based on their own anatomy. However, most studies use atlas derived ROI and hence it becomes necessary to evaluate these ROI derived from atlases compared with those derived from individual anatomy.

To the best of our knowledge there has been no study investigating sensitivity of the results of functional connectivity studies with respect to the shape and volume of the ROI used to sample brain areas of interest. Here we investigate one aspect of this issue: the variance of the shape and volume of the hippocampal ROI derived from three brain atlases: a frequency based brain atlas by Hammers et al [7] and two more widely used, single-subject atlases: AAL [6] and Brodmann areas defined in the PickAtlas toolbox $[4,5]$. In this study we used the extended version of the frequency atlas [7] based on manual delineations of 30 brains. The maximum probability map was obtained after co-registering all individual atlases into MNI space using the "Segment" module in SPM5 (http://www.fil.ion.ucl.ac.uk/spm/). We compare the atlas-derived ROIs with the results of the segmentation using an automatic algorithm, SACHA, implemented as part of the Brainvisa environment (http://brainvisa.info) [15]. 
Our interest to evaluate hippocampal ROI is explained by the importance of this structure in studies (especially ROI-based functional connectivity analisys of fMRI data) in patients with epilepsy and Alzheimer's disease $[1,2,16,17,18]$. 


\section{Materials \& Methods}

\section{Data}

Eighteen healthy subjects (five females; mean age 34.7 years and range: 25 - 56 years) were included. The criterion for inclusion into the study was absence of neurological pathology. All subjects gave written informed consent (Joint Ethics Committee of the National Hospital for Neurology and Neurosurgery and UCL Institute of Neurology).

High-resolution 3D T1-weighted MR images were acquired (Fast Spoiled Gradient Recalled [FSPGR]) on a 3T General Electric Excite HD scanner using a standard head coil: TR/TE/TI 8/3.1/450ms, flip angle $20^{\circ} ; 1561.1 \mathrm{~mm}$-thick coronal slices; matrix $256 \times 256 ; 24 \times 18 \mathrm{~cm}$ field of view; scan time 7 min.

\section{Data processing and analysis}

Individual ROIs for both hippocampi of each subject were segmented from the MR images using SACHA. The comparison of the atlas-based ROIs and individual SACHA-derived ROIs (further called as "individual ROIs") was performed in MNI space for two reasons: the fMRI analyses were to be performed in MNI space; all three atlases are available in MNI space. SACHA ROIs were evaluated by a trained observer (EW) for ensuring their consistency as a gold standard. T1-weighted images were transformed to MNI space using nonlinear warping as implemented in SPM5 [19]. The resulting transformation parameters were applied to the images of individual ROIs in order to register them to MNI space (voxel size in the template image is $2 \times 2 \times 2 \mathrm{~mm}$ ). The result of the registration was checked in the area of both hippocampi by visual evaluation by an expert-neuroanatomist (CK).

Using individual ROIs as the gold standard, the following volumetric and spatial correspondence measures were calculated as described in $[20,21]: \mathrm{RV}=$ the relative error on volume (the optimal value is $0 \%$ ); $\mathrm{K}=$ Dice overlap index, quantifying the proportion of properly classified voxels (the optimal value is $100 \%$ ); $\mathrm{FP}$ and $\mathrm{FN}=$ the proportions (in \% of total $\mathrm{ROI}$ volumes) of false positive and false negative voxels according to SACHA-based ROIs, respectively. In addition, the distance between the centre of the surface voxels of two ROIs is considered in three ways (indices measured in millimeters): the average symmetric distance on the whole boundary, Dm; the maximum of 
the symmetric distance (Hausdorff distance), DM; 95 percentile of DM, D95. The formulas for the indices can be found in the Appendix.

Two tailed t-test has been performed to test difference between mean values of the calculated indices comparing performance of (1) different atlases and (2) performance of right and left ROI within each atlas. 


\section{Results}

The results in the form of summary statistics of the calculated indices (mean, standard deviation, minimum and maximum values) are summarised in the table 1. Individual ROIs obtained for right hippocampus of subject \#8 overlaid over atlas-based ROIs are shown separately for each atlas on the same slices (Figure 1).

The values of the index describing the averaged similarity of the shape of the individual and atlas-derived ROI (Dm, in mm) show better performance of the Hammers atlas: for the right ROIs, AAL atlas $3.5(\mathrm{SD}=0.3)$, Hammers atlas $2.6(\mathrm{SD}=0.7)$, PickAtlas $3.5(\mathrm{SD}=0.4)$; for the left ROIs, AAL atlas $3.7(\mathrm{SD}=0.5)$, Hammers atlas $2.8(\mathrm{SD}=0.6)$, PickAtlas $3.6(\mathrm{SD}=0.9)$. However the values of the index describing the extreme deviation of the shape of the individual and atlas-derived ROI (DM, in $\mathrm{mm}$ ) show that the largest local error is to be found for Hammers atlas: for the right ROIs, AAL atlas 12 $(\mathrm{SD}=2)$, Hammers atlas 15.3 ( $\mathrm{SD}=2)$, PickAtlas $11(\mathrm{SD}=1.7)$; for the left ROIs, AAL atlas $12(\mathrm{SD}=1)$, Hammers atlas $16(\mathrm{SD}=2)$, PickAtlas $11.3(\mathrm{SD}=2.2)$.

Both two tailed t-test and non-parametric Wilcoxon rank test performed to reveal difference between mean values of the indices calculated for ROI from the three atlases showed highly significant $(\mathrm{p}<0.001)$ difference between mean values of the indices RV, K, FP, FN both for right and left ROI. There is no significant $(\mathrm{p}<0.001)$ difference between mean values of the indices Dm, DM, D95 for AAL atlas and PickAtlas (both for right and left ROI), whereas significant $(\mathrm{p}<0.001)$ difference is revealed for Hammers atlas compared with both other atlases.

A significant difference $(\mathrm{p}<0.01)$ between mean values for right and left ROI has been observed in the following indices: FN index for AAL atlas; RV, FP, FN indices for PickAtlas. No such difference was observed for any index for the Hammers atlas. 


\section{Discussion}

Using T1-weighted brain images from eighteen healthy volunteers, we performed spatial comparison between individually segmented hippocampal ROIs (done using software SACHA and checked by an expert as suitable to be considered as gold standard) and ROI derived from three brain atlases: Hammers et al's frequency based atlas, AAL atlas and the Brodmann areas available in the PickAtlas toolbox.

The AAL atlas contains ROIs defined manually on the high resolution MNI single-subject MRI brain template [6]. The PickAtlas [5] uses the MNI template for normalisation and probes the Talairach Daemon [4] across the entire Talairach space (created from a single hemisphere of a single subject) to generate tables based on coordinate position. In contrast, the frequency based atlas used in this work [7] was developed using multiple subjects in stereotaxic space. After manual segmentation in the individual space, the MRI volumes of the subjects were spatially normalised to T1-weighted MRI template in MNI/ICBM 152 space, as contained in the SPM5 package. This significant difference in the approaches applied to develop the three atlases chosen for this study along with differences in manual segmentation protocols used to define ROIs explain better volumetric correspondence of the individual ROIs and ROIs derived from the frequency based atlas (indices RV, K, FP), although with a greater false negative rate.

The three atlases chosen for our study rely on normalization of the individual brain to a stereotaxic space, a process that could contribute to the degradation of the segmentation results. Therefore, the high degree of spatial correspondence of the normalised individual T1-weighted images and MNI template in the area of both hippocampi was confirmed by an expert-neuronatomist after visual evaluation, which should always form part of ROI-based fMRI data analyses.

When taking into account the results of the comparison of ROIs performed in this study in the context of an ROI-based analysis of fMRI data, it is necessary to note that the comparison was performed in the space of the T1-weighted images warped to the MNI space. The fMRI data has to be warped to the same space in order to use both individual and atlas-based ROIs. The uncertainty of warping fMRI data has to be considered when specifying the required precision for the definition of ROIs. For example, in the case of hippocampal ROIs the fMRI data is prone to severe distortions in the 
area of interest causing increased uncertainty in the result of warping which may have a greater influence on the quality of the results than ROI definition. In this context it may appear that the frequency based atlas used in our study provides hippocampal ROIs with accuracy which is satisfactory for most studies as the observed difference in comparison with individual ROI (indices RV, K, FP, FN, $\mathrm{Dm})$ is of the same level of magnitude as the effect of uncertainties introduced while warping the fMRI data from individual to MNI space using up to date methods [22]. It is most likely that special investigation is necessary to conclude the same about hippocampal ROIs derived from PickAtlas and AAL atlas, as the accuracy of segmentation is very low (Table 1).

The low performance of Hammers atlas compare to other two atlases in DM and D95 indices is due to significant underestimation of the hippocampal tail which is detected by visual comparison of individual ROIs and ROIs derived from atlases.

The absence of the difference between mean values of the indices for right and left ROI observed in Hammers atlas as oppose to two other atlases (see Results) is indicative of better performance for the Hammers atlas and may reflect the fact that AAL atlas and PickAtlas are single-brain atlases and therefore are more subject to this type of bias.

In spite of the extreme underestimation, the ROI from PickAtlas might be satisfactory for the studies in which overestimation has to be avoided; in fact, false positive ration is lower for PickAtlas than for the other two atlases.

PickAtlas uses nonlinear transformation [23] to convert coordinates between MNI and Talairach spaces [5]. More precise transformation was suggested recently [24], which suggests that the accuracy of ROIs generated by PickAtlas toolbox may be increased.

This study was performed using data obtained from healthy volunteers and the results can not be extrapolated to cases with pathology or abnormal brains. However, one should generally assume that, in patients with hippocampal abnormalities (e.g. hippocampus sclerosis), the performance of any atlas will be much worse than in our study and that the levels of performance obtained here represent upper bounds. Therefore using individual segmentation (either automated or manual) is advisable in pathological cases. 


\section{Future work}

The sensitivity of functional connectivity estimates to ROI definition methods remains to be investigated. Our results suggest that ROI definition methodology can have a drastic influence on fMRI studies of hippocampal activity, with even greater impact in pathological cases. This highlights the direction for further work. 


\section{Conclusions}

The frequency based atlas [7] demonstrates higher accuracy for hippocampal segmentation than AAL atlas and PickAtlas in healthy volunteers. We recommend that the inclusion of erroneously classified voxels and exclusion of erroneously unclassified voxels must be carefully evaluated and measures taken

to minimise their impact on the sensitivity and specificity of the correlation studies (specifically ROIbased functional connectivity studies using fMRI data). 


\section{Acknowledgements}

This work was undertaken at UCLH/UCL who received a proportion of funding from the UK

Department of Health's NIHR Biomedical Research Centres funding scheme. Work funded through a grant from the Medical Research Council (MRC grant number G0301067). We are grateful to the Big Lottery Fund, Wolfson Trust and National Society for Epilepsy for supporting the NSE MRI scanner.

CK was funded through the Biotechnology associateship programme of the Department of Biotechnology, Government of India. 


\section{References}

1. Wagner K, Frings L, Halsband U, Everts R, Buller A, Spreer J, Zentner J, SchulzeBonhage A. Hippocampal functional connectivity reflects verbal episodic memory network integrity. Neuroreport. 2007;18(16):1719-23.

2. Bettus G, Guedj E, Joyeux F, Confort-Gouny S, Soulier E, Laguitton V, Cozzone PJ, Chauvel P, Ranjeva JP, Bartolomei F, Guye M. Decreased basal fMRI functional connectivity in epileptogenic networks and contralateral compensatory mechanisms. Hum Brain Mapp. 2008; [in press]

3. Chupin M, Hammers A, Bardinet E, Colliot O, Liu RS, Duncan JS, Garnero L, Lemieux L. Fully automatic segmentation of the hippocampus and the amygdala from MRI using hybrid prior knowledge. Med Image Comput Comput Assist Interv Int Conf Med Image Comput Comput Assist Interv. 2007;10(Pt 1):875-82

4. Lancaster JL, Woldorff MG, Parsons LM, Liotti M, Freitas CS, Rainey L, Kochunov PV, Nickerson D, Mikiten SA, Fox PT. Automated Talairach atlas labels for functional brain mapping. Hum. Brain Mapp. 2000; 10 (3): 120-131.

5. Maldjian JA, Laurienti PJ, Kraft RA, Burdette JH. An automated method for neuroanatomic and cytoarchitectonic atlas-based interrogation of fMRI data sets NeuroImage 2003; 19: 1233-1239.

6. Tzourio-Mazoyer N., Landeau, B., Papathanassiou, D., Crivello, F., Etard, O., Delcroix, $\mathrm{N}$. Automated anatomical labeling of activations in SPM using a macroscopic anatomical parcellation of the MNI MRI single subject brain. Neuroimage 2002; 15 : 273-289

7. Hammers A, Allom R, Koepp MJ, Free SL, Myers R, Lemieux L, Mitchell TN, Brooks DJ, Duncan JS. Three-dimensional maximum probability atlas of the human brain, with particular reference to the temporal lobe. Hum Brain Mapp. 2003 ;19(4):224-47.

8. Bohm, C., Greitz, T., Seitz, R., Eriksson, L. Specification and selection of regions of interest (ROIs) in a computerized brain atlas. J. Cereb. Blood Flow Metab. 1991; 11: A64-A68.

9. Collins, D.L., Holmes, C.J., Peters, T.M., Evans, A.C.. Automatic 3-D model-based neuroanatomical segmentation. Hum. Brain Mapp. 1995; 3:190-208.

10. Yasuno, F., Hasnine, A.H., Suhara, T., Ichimiya, T., Sudo, Y., Inoue, M., Takano, A., Ou, T., Ando, T., Toyama, H. Template-basedmethod for multiple volumes of interest of human brain PET images. NeuroImage 2002; 16: 577-586.

11. Hammers A, Koepp MJ, Free SL, Brett M, Richardson MP, Labbé C, Cunningham VJ, Brooks DJ, Duncan J. Implementation and application of a brain template for multiple volumes of interest. Hum Brain Mapp. 2002 ;15:165-74.

12. Poldrack R A Region of interest analysis for fMRI. Soc Cogn Affect Neurosci. 2007; 2: 67-70 doi:10.1093/scan/nsm006

13. Buck R, Singhal H, Arora J, Schlitt H, Constable R.T Detecting change in BOLD signal between sessions for atlas-based anatomical ROIs NeuroImage 2008; 40: 11571165

14. Mazziotta JC. Brain mapping: its use in patients with neurological disorders. Rev Neurol. 2001; 157(8-9 Pt 1):863-71

15. Cointepas, Y., Mangin, J.-F., Garnero, L., Poline, J.-B., and, H. BrainVISA: software platform for visualization and analysis of multimodality brain data. HBM-2001, p. S98.

16. Zhang HY, Wang SJ, Xing J, Liu B, Ma ZL, Yang M, Zhang ZJ, Teng GJ. Detection of PCC functional connectivity characteristics in resting-state fMRI in mild Alzheimer's disease. Behav Brain Res 2008; [in press] 
17. Zhou Y, Dougherty JH Jr, Hubner KF, Bai B, Cannon RL, Hutson RK. Abnormal connectivity in the posterior cingulate and hippocampus in early Alzheimer's disease and mild cognitive impairment. Alzheimers Dement. 2008; 4(4):265-70.

18. Carmichael OT, Aizenstein HA, Davis SW, Becker JT, Thompson PM, Meltzer CC, Liu Y. Atlas-based hippocampus segmentation in Alzheimer's disease and mild cognitive impairment. Neuroimage. 2005 Oct 1;27(4):979-90

19. Ashburner J, Friston KJ. Unified segmentation. Neuroimage. 2005 Jul 1;26(3):839-51

20. Chupin M, Mukuna-Bantumbakulu A R, Hasboun D, Bardinet E, Baillet S, Kinkingnéhun S, Lemieux L, Dubois B, Garnerob L Anatomically constrained region deformation for the automated segmentation of the hippocampus and the amygdala: Method and validation on controls and patients with Alzheimer's disease Neurolmage 2007; 34 : 996-1019

21. Gerig, G., Jomier, M., Chakos, M., 2001. Valmet: a new validation tool for assessing and improving 3D image segmentation. MICCAI 2001, vol. 2208, pp. 516-523

22. Frackowiak, R.S.J., Friston, K.J., Frith, C., Dolan, R., Price, C.J., Zeki, S., Ashburner, J., Penny, W.D. Human Brain Function, 2nd edition. 2003 Academic Press

23. Brett M, Christoff K, Cusack R, Lancaster J. Using the Talairach atlas with the MNI template. NeuroImage 2001, 13:S85.

24. Lancaster JL, Tordesillas-Gutiérrez D, Martinez M, Salinas F, Evans A, Zilles K, Mazziotta JC, Fox PT. Bias between MNI and Talairach coordinates analyzed using the ICBM-152 brain template. Hum Brain Mapp. 2007 Nov;28(11):1194-205. 


\section{Appendix}

The segmentation quality indices compare the ROI from each atlas, Seg, with the standard ROI obtained by SACHA, Ref. Seven indices were used to quantify the accuracy of the method and facilitate comparison with published values [20,21].

$\mathrm{RV}$ is the relative error on volume; it expresses the difference in volume of segmented object

$\mathrm{O}_{\text {Seg }}$ and reference object $\mathrm{O}_{\text {Ref }}$, relatively to their average:

$$
R V\left(O_{\text {Seg }}, O_{\mathrm{Re} f}\right)=2 \frac{\left|V_{O_{\text {Seg }}}-V_{O_{\mathrm{Re} f}}\right|}{V_{O_{\text {Seg }}}+V_{O_{\mathrm{Re} f}}}
$$

The optimal value for this index, consistent with perfect agreement, is $0 \%$.

Index $\mathrm{K}$ characterises overlap between $\mathrm{O}_{\mathrm{Seg}}$ and $\mathrm{O}_{\mathrm{Ref}}$, describing the number of properly classified voxels, without taking into account the number of ill-classified voxels:

$$
K\left(O_{S e g}, O_{\operatorname{Re} f}\right)=2 \frac{V_{O_{S e g} \cap O_{\operatorname{Re} f}}}{V_{O_{S e g}}+V_{O_{\operatorname{Re} f}}}
$$

The optimal value is $100 \%$.

The numbers of false positives, FP, and false negatives, FN, are computed here relatively to the number of voxels labelled as $\mathrm{O}_{\text {Seg }}$ or $\mathrm{O}_{\text {Ref: }}$ :

$$
\begin{aligned}
& F P\left(O_{S e g}, O_{\operatorname{Re} f}\right)=\frac{V_{O_{S e g}}-V_{O_{S e g} \cup O_{\operatorname{Re} f}}}{V_{O_{S e g} \cap O_{\operatorname{Re} f}}} \\
& F N\left(O_{S e g}, O_{\operatorname{Re} f}\right)=\frac{V_{O_{\operatorname{Re} f}}-V_{O_{S e g} \cap O_{\operatorname{Re} f}}}{V_{O_{S e g} \cup O_{\operatorname{Re} f}}}
\end{aligned}
$$

The local behaviour on the boundary can be characterised by its surface voxels (defined as the voxels of $\mathrm{O}$ with at least one 26-neighbour outside of $\mathrm{O}$ ). The distance between the centre of the surface voxels of $\mathrm{O}_{\mathrm{Seg}}$ and those of $\mathrm{O}_{\mathrm{Ref}}$ is considered in three ways. First, the average symmetric distance on the whole boundary, Dm, is computed:

$$
\operatorname{Dm}\left(O_{\operatorname{Seg}}, O_{\operatorname{Re} f}\right)=\max \left[h\left(O_{\operatorname{Seg}}, O_{\operatorname{Re} f}\right), h\left(O_{\operatorname{Re} f}, O_{\operatorname{Seg}}\right)\right\rfloor
$$

where 
$h(A, B)=\frac{1}{N_{A}} \sum_{a \in A} d(a, B)$

Second, the maximum of the symmetric distance (Hausdorff distance), DM, is considered:

$D M\left(O_{\text {Seg }}, O_{\operatorname{Re} f}\right)=\max \left[H\left(O_{S e g}, O_{\operatorname{Re} f}\right), H\left(O_{\operatorname{Re} f}, O_{\operatorname{Seg}}\right)\right]$

where

$H(A, B)=\max _{a \in A}[d(a, B)]$

with d the Euclidian distance.

The last index is used to discard sporadic errors, by considering the distance which explains the 95 percentile of DM, and it was called D95. All distances are expressed in millimetres. 
Figure 1. Fragments of sagittal, coronal and axial projections of T1 weighted image showing individual ROI (subject 8) and ROI derived from (a) frequency based atlas, (b) AAL atlas, (c) PickAtlas. The same slices are shown in all three cases. The red colour in encodes voxels covered only by individual ROI, green - voxels covered only ROI from one of the atlases, yellow - voxels in the area of overlap between the individual ROI and ROI from an atlas. 
Table 1. Segmentation quality indices for the three atlases. $V_{\text {ref }}-$ volume of the individual ROI, V - volume of the ROIs from the atlases. The explanation of the indices is in the Appendix. The index values averaged across all subjects are presented in the format: mean \pm standard deviation (minimum-maximum).

\begin{tabular}{|c|c|c|c|c|}
\hline ROI & Index & Hammers atlas & AAL atlas & PickAtlas \\
\hline \multirow{5}{*}{ Right } & $\mathrm{V}_{\text {ref }}\left(\mathrm{cm}^{3}\right)$ & $3.7 \pm 0.4(3.1-4.3)$ & $3.7 \pm 0.4(3.1-4.3)$ & $3.7 \pm 0.4(3.1-4.3)$ \\
\cline { 2 - 5 } & $\mathrm{V}\left(\mathrm{cm}^{3}\right)$ & 3 & 7.6 & 1 \\
\cline { 2 - 5 } & $\mathrm{RV}(\%)$ & $19.7 \pm 9.4(2.6-35.9)$ & $69.3 \pm 8.3(54.4-84.1)$ & $116 \pm 6.3(104-126)$ \\
\cline { 2 - 5 } & $\mathrm{K}(\%)$ & $68.5 \pm 5.6(56-74.7)$ & $43.8 \pm 5.8(29.7-53.3)$ & $35.5 \pm 2.9(27.7-40.2)$ \\
\cline { 2 - 5 } & $\mathrm{FP}(\%)$ & $16.4 \pm 3.5(8.7-20.5)$ & $58.1 \pm 3.2(51.7-63.8)$ & $4 \pm 1.5(0.8-5.8)$ \\
\cline { 2 - 5 } & $\mathrm{FN}(\%)$ & $31.3 \pm 5.5(21.8-43)$ & $13.7 \pm 3.8(9.7-25.3)$ & $74.4 \pm 2.2(70-78.6)$ \\
\cline { 2 - 5 } & $\mathrm{Dm}(\mathrm{mm})$ & $2.6 \pm 0.7(1.7-4.1)$ & $3.5 \pm 0.3(3-3.9)$ & $3.5 \pm 0.4(2.9-4.3)$ \\
\cline { 2 - 5 } & $\mathrm{DM}(\mathrm{mm})$ & $15.3 \pm 2.1(10.8-19.3)$ & $12 \pm 2(9.2-15.7)$ & $10.9 \pm 1.7(8.5-14)$ \\
\cline { 2 - 5 } & $\mathrm{D} 95(\mathrm{~mm})$ & $14.6 \pm 2(10.2-18.2)$ & $10.7 \pm 2(8.5-14.7)$ & $9.9 \pm 1.7(7.5-12.2)$ \\
\hline \multirow{5}{*}{ Left } & $\mathrm{V}_{\text {ref }}\left(\mathrm{cm}^{3}\right)$ & $3.3 \pm 0.4(2.4-4)$ & $3.3 \pm 0.4(2.4-4)$ & $3.3 \pm 0.4(2.4-4)$ \\
\cline { 2 - 5 } & $\mathrm{V}\left(\mathrm{cm}^{3}\right)$ & 2.7 & 7.5 & 1.1 \\
\cline { 2 - 5 } & $\mathrm{RV}(\%)$ & $21.4 \pm 10.5(1.8-39.4)$ & $77.4 \pm 11.1(60.1-104)$ & $98.4 \pm 10.4(71.4-113)$ \\
\cline { 2 - 5 } & $\mathrm{K}(\%)$ & $65.7 \pm 6.1(49.9-74.1)$ & $47.1 \pm 6(33.1-55.2)$ & $36.7 \pm 7(13.3-43.6)$ \\
\cline { 2 - 5 } & $\mathrm{FP}(\%)$ & $17.9 \pm 6.6(9.1-34.8)$ & $59.7 \pm 4.8(51.7-70.7)$ & $8.4 \pm 5.2(2.3-20.4)$ \\
\cline { 2 - 5 } & $\mathrm{FN}(\%)$ & $32.8 \pm 5.1(23.1-39.2)$ & $9.2 \pm 2.6(4.1-16.2)$ & $68.9 \pm 3.2(61.1-73.2)$ \\
\cline { 2 - 5 } & $\mathrm{Dm}(\mathrm{mm})$ & $2.8 \pm 0.6(1.6-4.5)$ & $3.7 \pm 0.5(3.1-4.6)$ & $3.6 \pm 0.9(2.7-6.5)$ \\
\cline { 2 - 5 } & $\mathrm{DM}(\mathrm{mm})$ & $16 \pm 2(11.7-20.7)$ & $11.9 \pm 1(10-14.7)$ & $11.3 \pm 2.2(8.5-19)$ \\
\cline { 2 - 5 } & $\mathrm{D} 95(\mathrm{~mm})$ & $15.3 \pm 2(10.8-20.4)$ & $10.6 \pm 1.1(8.9-13.4)$ & $10.3 \pm 2(8-17.2)$ \\
\hline
\end{tabular}

